\title{
Discontinuous Boundary Elements for Fluid Flow Problems in Discrete Fracture Networks
}

\author{
Bin Wang ${ }^{1,2}$ | Yin Feng*1 | Xu Zhou ${ }^{2}$ | Sandra \\ Pieraccini $^{3,6}$ | Stefano Scialò ${ }^{4,6}$ | Corrado Fidelibus ${ }^{5}$
}

\footnotetext{
${ }^{1}$ Department of Petroleum Engineering, University of Louisiana at Lafayette, Lafayette, Louisiana, USA

${ }^{2}$ Craft \& Hawkins Department of Petroleum Engineering, Louisiana State University, Baton Rouge, LA, USA

${ }^{3}$ Dipartimento di Ingegneria Meccanica e Aerospaziale, Politecnico di Torino, Torino, Italy

${ }^{4}$ Dipartimento di Scienze Matematiche, Politecnico di Torino, Torino, Italy

${ }^{5}$ Dipartimento di Ingegneria dell'Innovazione, Università del Salento, Lecce, Italy

${ }^{6}$ Member of INdAM-GNCS research group

\section{Correspondence}

Yin Feng, Department of Petroleum Engineering, University of Louisiana at Lafayette

Email: yin.feng@louisiana.edu

Funding information

Funder One, Board of Regents of the State of Louisiana, Grant/Award Numbers: LEQSF(2017-20)-RD-A-20; Funder Two, MIUR grant Dipartimenti di Eccellenza 2018-2022 (E11G18000350001) and MIUR project PRIN 201744KLJL_004; Funder Three, INdAM-GNCS
}

Modeling fluid flow in three-dimensional (3D) Discrete Fracture Networks (DFNs) is of relevance in many engineering applications, such as hydraulic fracturing, oil/gas production, geothermal energy extraction, nuclear waste disposal and $\mathrm{CO}_{2}$ sequestration. A new Boundary Element Method (BEM) technique with discontinuous quadratic elements and a parallel Domain Decomposition Method (DDM) is presented herein for the simulation of the steady-state fluid flow in 3D DFN systems with wellbores, consisting of planar fractures having arbitrary properties and wellbore trajectories. Numerical examples characterized by DFNs of increasing complexity are investigated to evaluate the accuracy and efficiency of the presented technique. The results show that accurate solutions can be obtained with less nodes than with mesh-based methods (e.g. Finite Element Method). In addition, the DDM algorithm used provides a quite fast convergence. The simulation results of the fluid flow around intersections among traces (linear intersections between fractures), intersections between traces and a fracture boundaries, and wellbore intersections is accurate.

Source code is available at

https://github.com/BinWang0213/PyDFN3D.

KEYWORDS

Discrete Fracture Network (DFN); fractured rock hydrology; 
Boundary Element Method (BEM); Domain Decomposition Method (DDM); subsurface fluid flow

\section{1 | INTRODUCTION}

Modeling fluid flow in fractured rocks has been for long of relevance in many engineering applications, such as hydraulic fracturing, oil/gas production, geothermal energy extraction, nuclear waste disposal and $\mathrm{CO}_{2}$ sequestration [31, 37, 30 . 32. 43]. Two predictive models are generally used: the Equivalent Porous Medium (EPM) and the Discrete Fracture Network (DFN). EPM is considered when fractures are highly connected and the fracture network can be condensed into a porous medium with associated permeability tensor 34 39]. DFN is alternative to EPM for multi-scale fracture networks where all the fractures must be explicitly represented. [30,26].

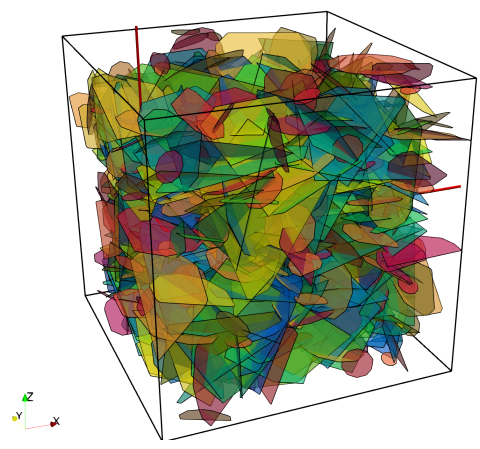

(a) Typical DFN with 932 fractures and a horizontal well

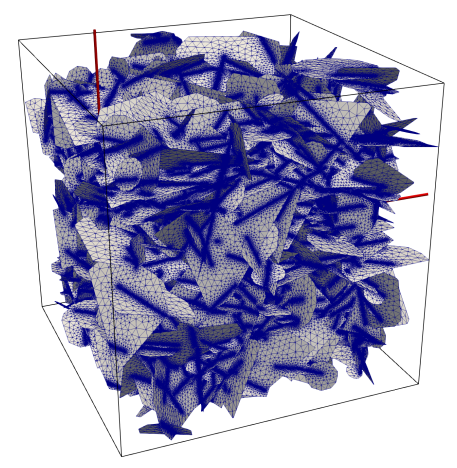

(b) FEM/FVM Mesh with 12.54 million

2D elements and 5.45 million nodes

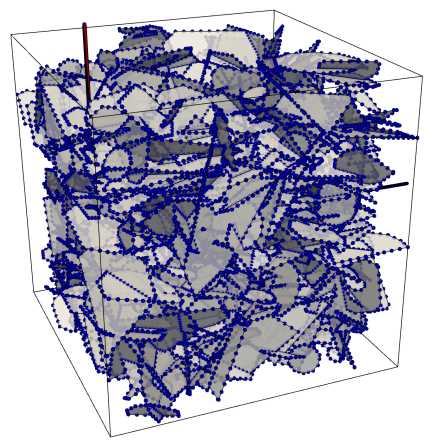

(c) BEM Mesh with 0.13 million 1D elements and 0.11 million nodes

FIGURE 1 A DFN example; comparison between FEM/FVM mesh and BEM mesh

In the last decades, significant efforts have been produced to simulate the fluid flow in three-dimensional (3D) DFNs. The adopted numerical methods can be categorized into: 1) mesh-based methods, 2) the Equivalent Pipe Network (EPN) and 3) the Boundary Element Method (BEM). In the mesh-based methods the computational mesh is firstly 
generated over the entire fracture system and the flow field is then obtained by resorting to the Finite Element Method (FEM) or the Finite Volume Method (FVM). These methods offer considerable precision but, given the geometrical complexity of a DFN (see Fig 1a] the use implies high computational demand and memory usage [28, also originating from high-quality mesh generation and Local Grid Refinement (LGR), both required to accurately resolve the sharp pressure gradients around the intersections (traces) among fractures and in the near-field of a well[26]. In Fig. 1b, an example of a DFN consisting of 932 fractures of various sizes and orientations is depicted; a relatively coarse FEM mesh has been built consisting of nearly 1.61 million nodes and 3.84 million triangular elements. In spite of the limited number of fractures of the network, the computational demand is huge and the FEM solution can be handled only through the use of sophisticated numerical techniques. In this respect, several advanced techniques have been recently developed and documented in literature. In 12 8 8 , 7, non-conforming meshes independently built on each fracture are used, and the minimization of a cost functional is applied to enforce a coupling of domain decomposition method and error estimators to control the accuracy of the solution. Techniques based on conforming polygonal meshes of complex DFNs have also been proposed, e.g. in [5.6 25], where the Virtual Element Method is used, in [4], by combining Mimetic Finite Differences and mixed finite elements, and in [15], where an Hybrid High Order method is applied.

In order to furnish a more efficient solution, the EPN method was alternatively proposed: the DFN is transformed into a network of one-dimensional conductors (pipes) connecting the mid-points of the traces in a fracture and having specific conductance [14 22. 47]. EPNs feature great advantages in terms of computational efficiency and simplicity and as such are suitable to model large fracture networks. However, unique pipe-connection patterns are difficult to generate and the equivalence gives rise to unavoidable errors. Recently, a graph-based method is proposed to reduce the error of EPNs 3.29.

The application of BEM requires a one-dimensional discretization ( $a$ mesh) of lines corresponding to the fracture edges and traces [41]; the method reveals being very accurate to resolve the sharp pressure gradients near traces and wells even when few one-dimensional elements are used, provided that semi-analytic basis functions are introduced for the representation of the variables 33 45]. In Fig. 1c, it is shown that the number of the boundary elements of the discretization is about two orders of magnitude less the number of elements associated to a mesh-based method.

The contributions concerning the use of BEM for the prediction of the fluid flow in three-dimensional DFNs are still quite limited. Shapiro and Andersson 40, 41,2] were the first to apply the method by resorting to constant boundary elements for fracture edges and traces. Later, Lenti and Fidelibus[22,35] proposed an advanced BEM technique in which quadratic elements are used for fracture edges and constant elements are used for internal traces. The choice of constant elements derived from the need to handle the flux discontinuities occurring around the intersections between traces in the plane of a fracture. An optimization procedure was also applied to reduce the memory usage when solving the global matrix. The authors indicated that the resort to low-order constant elements may lead to up to $22 \%$ flux balance error when compared to a reference solution. Yang et al [48] and Chen et al [18, 20, 19, 17] have recently proposed a BEM method to model transient shale gas flow problems in a 2D infinite domain where BEM with constant elements is used to solve matrix flow, finite difference method is used to solve 1D fracture flow within discrete fracture network and Laplace transform is used to resolve transient flow.

Fast solutions for the flow regime are crucial for industrial application of DFNs. In this sense, none of the three methods mentioned above prevails in offering such solutions. However, the scientific community may benefit from any contribution improving one of the methods. In this note, a novel BEM technique including discontinuous quadratic basis functions for the boundary elements and with the application of a parallel Domain Decomposition Method (DDM) is proposed for the prediction of the steady-state fluid flow in 3D DFNs. This technique efficiently resolves the problem at the intersections between traces, furnishes a solution of higher quality and allows handling fracture-wise homogeneous permeability and aperture (each fracture can have proper homogeneous values of permeability and aperture). 
The note is organized as follows: in Section 2, a detailed description of the adopted formulation and of the typical discretization associated to the proposed BEM technique is reported; in Section the DDM algorithm is briefly introduced and commented; in Section 4 the technique is tested and verified by using three verification examples and one synthetic field application example. Finally, the possible extensions of the technique are discussed and concluding remarks are drawn accordingly.

\section{2 | BEM FORMULATIONS}

In this section, with reference to the proposed technique, the BEM formulation adopted for the steady-state fluid flow in 3D DFNs is described. The derivation of the integral equations and of the final algebraic system are reported, given the special basis functions of the discontinuous elements.

\section{1 | Boundary integral equations}

With reference a local coordinate system $\mathrm{X}=\left\{X_{1}, X_{2}, X_{3}\right\}$, a three-dimensional fracture domain $\Omega^{\star}$ is considered. The mean plane of the fracture is $\pi$, whereas $\mathbf{n}_{\pi}$ and $t_{\pi}$ are the unit vector normal and tangent to $\pi$, respectively. A domain $\Omega$ is defined in $\pi$ and referred to a local coordinate system $\mathbf{x}=\left\{x_{1}, x_{2}\right\} ; \Omega$ is limited by the fracture edges; the boundary is $\Gamma$ with an outward normal $\mathbf{n}$. The domain $\Omega^{*}$ is such that $\Omega^{*}=\left\{X: X=\gamma+b_{f} \mathbf{t}_{\pi}, \gamma \in \pi\right\}$, being $b_{f}(\mathbf{x})$ the fracture thickness, or aperture, a number much smaller than each of the dimensions of $\Omega$ in $\pi$. In what follows $b_{f}(\mathbf{x})=b_{f}$ is assumed uniform in $\pi$, top and bottom walls of the fracture are planes parallel to $\pi$, thus the fluid velocities inside the fracture are parallel to $\pi$. In addition, it is also assumed that the relative distribution along $\mathbf{n}_{\pi}$ is uniform.

By assuming that the fracture intrinsic permeability $k$ (unit $\mathrm{m}^{2}$ ) is homogeneous and uniformly distributed in $\Omega^{\star}$ and the fluid dynamic viscosity $\mu$ (unit $\mathrm{kg} \mathrm{m}^{-1} \mathrm{~s}^{-1}$ ) is independent of pressure $p$, the governing equation for a steady-state fluid flow in $\Omega^{\star}$ with a generic volumetric source term $Q(\mathbf{X})$ (unit s ${ }^{-1}$ ) can be expressed as follows 16 :

$$
-\frac{k}{\mu} \nabla^{2} p(\mathbf{X})=Q(\mathbf{X})
$$

where $p(\mathbf{X})$ is fluid pressure (unit $\mathrm{kg} \mathrm{m}^{-1} \mathrm{~s}^{-2}$ ). The 3D problem can be reduced into a 2D problem by averaging Eq. 1 . along $\mathbf{n}_{\pi}$. By splitting the operator $\nabla$ in an in-plane component $\nabla_{\pi}$ and a normal component $\nabla_{\mathbf{n}_{\pi}}$ one has:

$$
\begin{gathered}
-\frac{k}{\mu} \nabla_{\pi}^{2} \int_{-\frac{b_{f}}{2}}^{\frac{b_{f}}{2}} p(\mathbf{X}) \mathrm{d} \mathbf{n}_{\pi}=\int_{-\frac{b_{f}}{2}}^{\frac{b_{f}}{2}} Q(\mathbf{X}) \mathrm{d} \mathbf{n}_{\pi} \\
-\frac{k b_{f}}{\mu} \nabla_{\pi}^{2} \tilde{p}(\mathbf{x})=q(\mathbf{x})
\end{gathered}
$$

in which $\tilde{p}(\mathbf{x})=\frac{1}{b_{f}} \int_{-b_{f} / 2}^{b_{f} / 2} p(\mathbf{X}) \mathrm{d} \mathbf{n}_{\pi}$ is the averaged pressure along $\mathbf{n}_{\pi}, q(\mathbf{x})=\frac{1}{b_{f}} \int_{-b_{f} / 2}^{b_{f} / 2} Q(\mathbf{X}) \mathrm{dn}_{\pi}$ is a distributed areal source. In what follows, $\nabla$ is used for $\nabla_{\pi}$ and $p$ is assimilated to $\tilde{p}$.

The fracture can be intercepted by other fractures, whose mean planes give rise to linear intersections (traces) in $\pi$. Also wellbores can cross the fracture, and the intersections are considered in what follows punctual point intersections (wellbore intersections) in $\pi$.

Assuming the rock matrix is impervious, there is no leakage from the blocks bounded by the fractures, therefore there are no areal sources, rather the source term $q(\mathbf{x})$ is given by linear sources and point sources [16] from the traces and the wellbore intersections, respectively. In what follows, the compound of traces in $\Omega$ is denoted by $t$, whereas 
$T$ is the set of all the traces in the same domain; wellbore intersections are denoted by $s$ in a set $S$ of all the wellbore intersections in $\Omega$. Therefore, the source term $q(\mathbf{x})$ can be written as:

$$
q(\mathbf{x})=\sum_{t \in T} \delta_{t, q_{t}}+\sum_{s \in S} q_{s} \delta_{s}
$$

being $\delta_{t, q_{t}}$ the Dirac Delta function defined, for any sufficiently regular function $\varphi(\mathbf{x})$, as $\left\langle\delta_{t, q_{t}}, \varphi\right\rangle=\int_{t} q_{t}(\gamma) \varphi(\gamma) d \gamma$, where $q_{t}(\gamma)$ (unit $\mathrm{m}^{2} \mathrm{~s}^{-1}$ ), is the concentrated volumetric source per unit trace length on $t$, whereas $\delta_{s}$ for wellbore $s \in S$ is the Dirac Delta function located at the intersection point between the well and the mean fracture plane, $\mathbf{x}_{s}$, defined as $\left\langle\delta_{s}, \varphi\right\rangle=\varphi\left(\mathbf{x}_{s}\right)$ and $q_{s}\left(\right.$ unit $\left.\mathrm{m}^{3} \mathrm{~s}^{-1}\right)$ is the volumetric point source at wellbore $s$.

By substituting Eq. 3 into Eq. 2] the governing equation for the fluid flow in a fracture is obtained [23]:

$$
-\frac{k b_{f}}{\mu} \nabla^{2} p=\sum_{t \in T} \delta_{t, q_{t}}+\sum_{s \in S} q_{s} \delta_{s}
$$

Dirichlet and Neumann boundary conditions apply to the boundary $\Gamma$ for the solution of Equation [4]. The Boundary Integral Equation (BIE) corresponding to Eq. (4) is as follows:

$$
\begin{array}{r}
-\frac{k b_{f}}{\mu}\left(c\left(\mathbf{x}_{i}\right) p\left(\mathbf{x}_{i}\right)+\int_{\Gamma} p(\mathbf{x}) \frac{\partial w\left(\mathbf{x}_{i}, \mathbf{x}\right)}{\partial \mathbf{n}} \mathrm{d} \Gamma\right)=-\frac{k b_{f}}{\mu} \int_{\Gamma} \frac{\partial p}{\partial \mathbf{n}}(\mathbf{x}) w\left(\mathbf{x}_{i}, \mathbf{x}\right) \mathrm{d} \Gamma \\
-\sum_{t \in T} \int_{t} q_{t}(\mathbf{x}) w\left(\mathbf{x}_{i}, \mathbf{x}\right) \mathrm{d} t-\sum_{s \in S} q_{s} w\left(\mathbf{x}_{i}, \mathbf{x}_{s}\right)
\end{array}
$$

where the derivative of $p$ with respect to the normal is included, and where $\mathbf{x}_{i}, \mathbf{x}$ are a source point and a field point, respectively, $\mathbf{x}_{\mathbf{i}} \in \Omega$, including $\Gamma, \mathbf{x} \in \Gamma \cup T \cup S . c\left(\mathbf{x}_{i}\right)$ is a geometrical free term and $w$ is a weighing function.

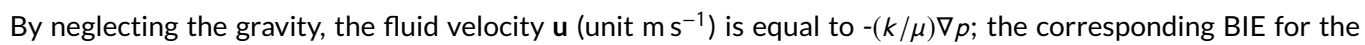
components $u_{j}\left(\mathbf{x}_{i}\right)$ is:

$$
\begin{array}{r}
c\left(\mathbf{x}_{i}\right) u_{j}\left(\mathbf{x}_{i}\right) b_{f}-\frac{k b_{f}}{\mu} \int_{\Gamma} p(\mathbf{x}) \frac{\partial}{\partial x_{j}}\left(\frac{\partial w\left(\mathbf{x}_{i}, \mathbf{x}\right)}{\partial \mathbf{n}}\right) \mathrm{d} \Gamma=-\frac{k b_{f}}{\mu} \int_{\Gamma} \frac{\partial p}{\partial \mathbf{n}}(\mathbf{x}) \frac{\partial w\left(\mathbf{x}_{i}, \mathbf{x}\right)}{\partial x_{j}} \mathrm{~d} \Gamma \\
-\sum_{t \in T} \int_{t} q_{t}(\mathbf{x}) \frac{\partial w\left(\mathbf{x}_{i}, \mathbf{x}\right)}{\partial x_{j}} \mathrm{~d} t-\sum_{s \in S} q_{s} \frac{\partial w\left(\mathbf{x}_{i}, \mathbf{x}_{s}\right)}{\partial x_{j}}
\end{array}
$$

Details on derivation and notation for the above BIEs are given in Appendix A. The weighing function $w$ corresponds to the fundamental solution of the steady-state fluid flow equation for a source point $\mathbf{x}_{i}$. The function $w$ and the derivative $\frac{\partial w}{\partial \mathbf{n}}$ with respect to the normal $\mathbf{n}$ are 13 :

$$
\begin{aligned}
w\left(\mathbf{x}_{i}, \mathbf{x}\right) & =\frac{1}{2 \pi} \ln \frac{1}{r\left(\mathbf{x}_{i}, \mathbf{x}\right)} \\
\frac{\partial w\left(\mathbf{x}_{i}, \mathbf{x}\right)}{\partial \mathbf{n}} & =\frac{1}{2 \pi} \frac{\left(\mathbf{x}_{i}-\mathbf{x}\right) \cdot \mathbf{n}}{r^{2}\left(\mathbf{x}_{i}, \mathbf{x}\right)}
\end{aligned}
$$

where $r\left(\mathbf{x}_{i}, \mathbf{x}\right)$ is the distance between $\mathbf{x}_{i}$ and $\mathbf{x}$. 


\section{2 | Discretization with discontinuous quadratic elements}

Lenti and Fidelibus [35] proposed a BEM technique for DFNs in which constant basis functions for the traces were used in order to deal with the flux discontinuities at trace-trace and trace-boundary intersections. However, due to the low accuracy of the constant element approximation, the application of the technique implies errors increasing with the geometrical complexity of the DFN. In this note, an advancement is proposed by resorting to quadratic basis functions and discontinuous Boundary Elements (BEs), intrinsically including flux discontinuities at the nodes. As shown in Fig. 2

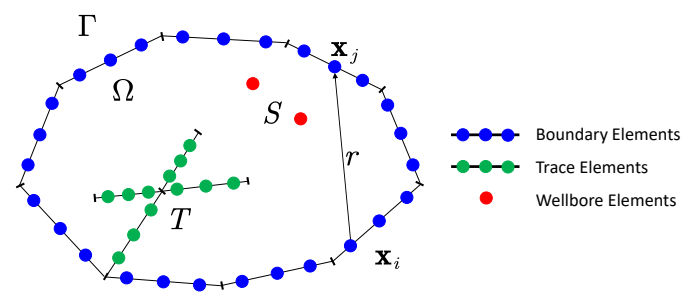

FIGURE 2 BEM discretization for a fracture domain; $\mathbf{x}_{i}$ is a collocation node, $\mathbf{x}_{j}$ an integration node; green, blue and red dots represent the collocation nodes.

a discretization is produced by subdividing the boundary $\Gamma$ and the traces $t \in T$ in straight BEs, and collocation nodes are placed on each element, coinciding with source points of Eq.5. Given the quadratic basis functions, three nodes are placed along each BE. It is to note that the nodes never coincide with trace-trace intersections and trace-boundary intersections, where the flux of $p$ is discontinuous. Other nodes can coincide with the wellbore intersections $s \in S$.

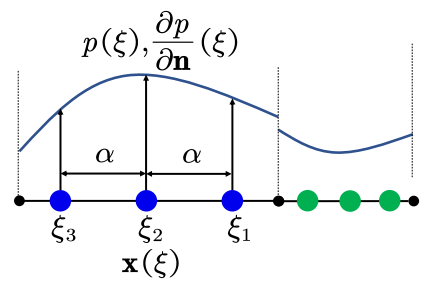

FIGURE 3 Discontinuous quadratic element used for boundary and trace elements, with $-1 \leqslant \xi \leqslant 1$, $\xi_{1}=-\alpha, \xi_{2}=0, \xi_{3}=\alpha$.

Being $\mathbf{N}=\left\{N_{i}\right\}_{i=1, \ldots, 3}$ the (elemental) quadratic basis functions (see Fig 3, pressure $p$ can be approximated as follows:

$$
p(\xi)=\sum_{i=1}^{3} p_{i} N_{i}(\xi), \quad \frac{\partial p}{\partial \mathbf{n}}(\xi)=\sum_{i=1}^{3}\left(\frac{\partial p}{\partial \mathbf{n}}\right)_{i} N_{i}(\xi), \quad-1 \leqslant \xi \leqslant 1
$$

where $\xi$ is a local normalized coordinate, $p(\xi), \frac{\partial p}{\partial \mathbf{n}}(\xi)$ are defined in a finite dimensional space, $p_{i},\left(\frac{\partial p}{\partial \mathbf{n}}\right)_{i}$ are nodal values. Functions $\mathbf{N}$ are defined as follows:

$$
N_{1}=\frac{1}{2} \frac{\xi}{\alpha}\left(\frac{\xi}{\alpha}-1\right), N_{2}=\left(1-\frac{\xi}{\alpha}\right)\left(1+\frac{\xi}{\alpha}\right), N_{3}=\frac{1}{2} \frac{\xi}{\alpha}\left(\frac{\xi}{\alpha}+1\right), \quad 0<\alpha \leq 1
$$


where $\alpha$ is the collocation factor. For $0<\alpha<1$, the first and the third nodes of the element are shifted inside it of a normalized quantity equal to $\alpha$. For $\alpha=1$ the ordinary quadratic functions are obtained. A value of $\alpha=0.67$ is suggested [24] and assumed in the following. Boundaries and traces are approximated by using the same functions $\mathbf{N}$, using $\mathbf{x}=\left.\sum_{i=1}^{3} \mathbf{x}_{i} N_{i}(\xi)\right|_{\alpha=1}$, i.e. the shape functions of Eq. 9 with $\alpha=1$.

By denoting $J_{\text {tot }}$ the set of the indexes of all the nodes, a global numbering is introduced for the nodes and for the (global) basis functions $N_{i}$, being $N_{i}\left(\mathbf{x}_{j}\right)$ equal to 1 if and only if $i=j$, for $i, j \in J_{\text {tot }}$. The following sets are also introduced: $J_{\Gamma}$, including the indexes of the nodes belonging to the boundary $\Gamma ; J_{t}$, including the indexes of the nodes belonging to trace $t \in T ; J_{s}$, including the index of the node matching $\mathbf{x}_{s}$. The following sets are further defined; $J_{T}=\bigcup_{t \in T} J_{t}$; $J_{S}=\bigcup_{s \in S} J_{S}$. Without loss of generality the indexes of these sets are numbered consecutively, first counting the nodes on the boundary $\Gamma$ and then those on traces and wellbore intersections.

In what follows $p^{\Gamma}(\mathbf{x})=\sum_{j \in J_{\Gamma}} p_{j}^{\Gamma} N_{j}(\mathbf{x}), p^{T}(\mathbf{x})=\sum_{j \in J_{T}} p_{j}^{T} N_{j}(\mathbf{x}), p^{S}(\mathbf{x})=\sum_{j \in J_{S}} p_{j}^{S} N_{j}(\mathbf{x})$ are the discrete solutions on $\Gamma$, on the traces and on the wellbore intersections, respectively. Similarly, the discrete counterpart of $\frac{\partial p}{\partial \mathbf{n}}$ on $\Gamma$ is introduced as $\left(\frac{\partial p}{\partial \mathbf{n}}\right)^{\Gamma}(\mathbf{x})=\sum_{j \in J_{\Gamma}}\left(\frac{\partial p}{\partial \mathbf{n}}\right)_{j}^{\Gamma} N_{j}(\mathbf{x})$, whereas the source on trace $t \in T$ is denoted by $q_{t}^{t}(\mathbf{x})=\sum_{j \in J_{t}} q_{j}^{t} N_{j}(\mathbf{x})$. Finally, $\mathbf{p}^{\star}, \star=\{\Gamma, T, S\}$ is the array collecting column-wise the unknowns $\mathbf{p}_{j}^{\star}, j \in J_{\star},\left(\frac{\partial \mathbf{p}}{\partial \mathbf{n}}\right)^{\Gamma}$ collects the unknown derivatives of $p, \mathbf{q}^{T}$ collects $q_{j}^{t}$, for $j \in J_{t}$ and $t \in T$, and $\mathbf{q}^{S}$ collects $q_{s}, s \in S$.

By inserting the above definitions into the BIE Eq.(5) yields:

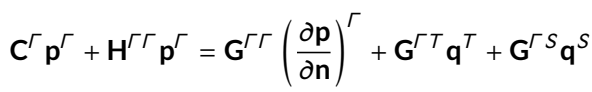

$$
\begin{aligned}
& \mathbf{C}^{T} \mathbf{p}^{T}+\mathbf{H}^{T \Gamma} \mathbf{p}^{\Gamma}=\mathbf{G}^{T \Gamma}\left(\frac{\partial \mathbf{p}}{\partial \mathbf{n}}\right)^{\Gamma}+\mathbf{G}^{T T} \mathbf{q}^{T}+\mathbf{G}^{T S} \mathbf{q}^{S} \\
& \mathbf{C}^{S} \mathbf{p}^{S}+\mathbf{H}^{S \Gamma} \mathbf{p}^{\Gamma}=\mathbf{G}^{S \Gamma}\left(\frac{\partial \mathbf{p}}{\partial \mathbf{n}}\right)^{\Gamma}+\mathbf{G}^{S T} \mathbf{q}^{T}+\mathbf{G}^{S S} \mathbf{q}^{S}
\end{aligned}
$$

where $\mathbf{C}^{\star}, \star=\{\Gamma, T, S\}$ are elementary matrices, with $\mathbf{C}_{i i}^{\star}$ equal to $\frac{k b_{f}}{\mu}$ when $\mathbf{x}_{i}$ is inside the domain and equal to $\frac{k b_{f}}{2 \mu}$ when $\mathbf{x}_{i}$ is on the boundary, and the $G$-type and $H$-type matrices contain the integrals in the same equation. In formula:

$$
\begin{aligned}
& G_{i j}^{\Gamma \Gamma}=\frac{k b_{f}}{\mu} \int_{\Gamma} w\left(\mathbf{x}_{i}, \mathbf{x}\right) N_{j}(\mathbf{x}) \mathrm{d} \Gamma, \quad i, j \in J_{\Gamma} ; \\
& G_{i j}^{\Gamma T}=\int_{t} w\left(\mathbf{x}_{i}, \mathbf{x}\right) N_{j}(\mathbf{x}) \mathrm{d} t, \quad i \in J_{\Gamma}, j \in J_{t}, t \in T ; \\
& G_{i j}^{\Gamma S}=w\left(\mathbf{x}_{i}, \mathbf{x}\right) N_{j}(\mathbf{x}), \quad i \in J_{\Gamma}, j \in J_{S} ; \\
& G_{i j}^{T \Gamma}=\frac{k b_{f}}{\mu} \int_{\Gamma} w\left(\mathbf{x}_{i}, \mathbf{x}\right) N_{j}(\mathbf{x}) \mathrm{d} \Gamma, \quad i \in J_{t}, j \in J_{\Gamma}, t \in T \\
& G_{i j}^{T T}=\int_{t} w\left(\mathbf{x}_{i}, \mathbf{x}\right) N_{j}(\mathbf{x}) \mathrm{d} t, \quad i, j \in J_{t}, t \in T ; \\
& G_{i j}^{T S}=w\left(\mathbf{x}_{i}, \mathbf{x}\right) N_{j}(\mathbf{x}), \quad i \in J_{T}, j \in J_{S} ; \\
& G_{i j}^{S \Gamma}=\frac{k b_{f}}{\mu} \int_{\Gamma} w\left(\mathbf{x}_{i}, \mathbf{x}\right) N_{j}(\mathbf{x}) \mathrm{d} \Gamma, \quad i \in J_{S}, j \in J_{\Gamma} ; \\
& G_{i j}^{S T}=\int_{t} w\left(\mathbf{x}_{i}, \mathbf{x}\right) N_{j}(\mathbf{x}) \mathrm{d} t, \quad i \in J_{S}, j \in J_{T} ; \\
& G_{i i}^{S S}=\frac{1}{2 \pi} \ln r_{S(i)}, \quad i \in J_{S} ; \\
& G_{i j}^{S S}=w\left(\mathbf{x}_{i}, \mathbf{x}\right), \quad i \neq j \in J_{S} ;
\end{aligned}
$$


Matrices $H^{\Gamma \Gamma}, H^{S \Gamma}, H^{T \Gamma}$ are defined like the G-type matrices $G^{\Gamma \Gamma}, G^{S \Gamma}, G^{T \Gamma}$ by substituting in the formulas the fundamental solution with the derivative with respect to the normal. Note that in general $G^{T \Gamma} \neq\left(G^{\Gamma T}\right)^{\top}, G^{S T} \neq$ $\left(G^{T S}\right)^{\top}, G^{S \Gamma} \neq\left(G^{\Gamma S}\right)^{\top}$. The diagonal entries of $G^{S S}$ goes to infinity for the distance $r\left(\mathbf{x}_{i}, \mathbf{x}\right)$ approaching to zero. Also, in order to avoid the singular point and apply the constant pressure boundary condition for wellbores, the nodes of pressure unknowns $p^{S}(\mathbf{x})$ are placed on the boundary of the borehole, thus having a distance $r_{s}$ from the collocation node at the centre of the well. This handling of the wellbores was used and validated in a previous work [45].

Eq. 10] can be expressed in compact form as follows:

$$
\left[\begin{array}{ccc}
\mathbf{C}^{\Gamma}+\mathbf{H}^{\Gamma \Gamma} & 0 & 0 \\
\mathbf{H}^{T \Gamma} & \mathbf{C}^{T} & 0 \\
\mathbf{H}^{S \Gamma} & 0 & \mathbf{C}^{S}
\end{array}\right]\left\{\begin{array}{c}
\mathbf{p}^{\Gamma} \\
\mathbf{p}^{T} \\
\mathbf{p}^{S}
\end{array}\right\}=\left[\begin{array}{lll}
\mathbf{G}^{\Gamma \Gamma} & \mathbf{G}^{\Gamma T} & \mathbf{G}^{\Gamma S} \\
\mathbf{G}^{T \Gamma} & \mathbf{G}^{T T} & \mathbf{G}^{T S} \\
\mathbf{G}^{S \Gamma} & \mathbf{G}^{S T} & \mathbf{G}^{S S}
\end{array}\right]\left\{\begin{array}{c}
(\partial \mathbf{p} / \partial \mathbf{n})^{\Gamma} \\
\mathbf{q}^{T} \\
\mathbf{q}^{S}
\end{array}\right\}
$$

On each node of the boundary, pressure $p$ or flux $\partial p / \partial n$ can be specified, for a Dirichlet or a Neumann condition, respectively, thus, the columns of the matrices in Eq.12]can be re-ordered to have the coefficients of all the unknowns on the left-hand side and the coefficients of all the known terms on the right-hand side. However, the prevailing condition is the insulation condition, i.e. $\partial p / \partial n=0$. Once the solution of Eq. 12 is obtained, pressure and velocity at any point $\mathbf{x}_{i}$ can be calculated by applying Eqs. 5.6

It is to remark that if a wellbore with a flow rate $q_{w}$ penetrates multiple fracture planes, thus forming, say, $s_{1}, s_{2}, \ldots, s_{n}$ punctual point intersections, one for each fracture, additional conditions have to be applied: same pressure value $p_{w}$ for all the wellbore intersections and $q_{s_{1}}+q_{s_{2}}+\ldots+q_{s_{n}}=q_{w}$. For example, if an injection well penetrates two fractures with given flow rate $q_{w}$, three unknowns and three equations are introduced for the wellbore. The three unknowns associated to the well are: the same wellbore pressure $p_{w}$ for both $s_{1}$ and $s_{2}$, the flow rates $q_{s_{1}}$ at fracture 1 and $q_{s_{2}}$ at fracture 2, respectively. Only two equations from the equation system 12 are available, thus a additional equation $q_{s_{1}}+q_{s_{2}}=q_{w}$ is required.

\section{3 | Fast analytical BIE integration}

After the BEM discretization is performed, given the singularity of the fundamental solution $w$, there is the need to perform singular integrations, nearly-singular integrations and non-singular integrations. The singular and nearly-singular integrations require special techniques, because the standard numerical integration may lead to large errors [33]. Element subdivision, analytical and semi-analytical integration, adaptive Gaussian quadrature, coordinate transformation and BIE modification 36.4427 can be adopted. Also exact analytical integration formulations are available[24,50 49. In this note, an exact integration formulation for discontinuous quadratic elements is derived based on the method proposed by [50]. The expressions for the integrals of $G$-type and $H$-type matrices for a boundary or a trace integration element in Eq. 11 are reported in what follows; the quantities $A_{l}, D_{l}, E_{l}, F_{l}, I_{l}, S_{l}, T_{l}(I=0, \ldots, 2)$ and $a, e$ are defined in Appendix B.

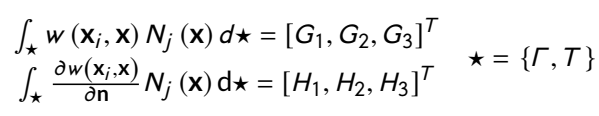


When a collocation node does not pertain to the integration element, the analytical integration formulations are:

$$
\begin{aligned}
& G_{1}=\frac{1}{2 \pi} \frac{J}{4}\left(\frac{A_{2}}{\alpha^{2}}-\frac{A_{1}}{\alpha}\right), G_{2}=\frac{1}{2 \pi} \frac{\jmath}{2}\left(A_{0}-\frac{A_{2}}{\alpha^{2}}\right), G_{3}=\frac{1}{2 \pi} \frac{\jmath}{4}\left(\frac{A_{2}}{\alpha^{2}}+\frac{A_{1}}{\alpha}\right) \\
& H_{1}=\frac{1}{2 \pi} \frac{e}{2}\left(\frac{F_{2}}{\alpha^{2}}-\frac{F_{1}}{\alpha}\right), H_{2}=\frac{1}{2 \pi} e\left(F_{0}-\frac{F_{2}}{\alpha^{2}}\right), H_{3}=\frac{1}{2 \pi} \frac{e}{2}\left(\frac{F_{2}}{\alpha^{2}}+\frac{F_{1}}{\alpha}\right)
\end{aligned}
$$

where $J$ is the Jacobian. When a collocation node is instead on the integration element, the formulations are:

$$
\begin{gathered}
G_{1}=\frac{1}{2 \pi} \frac{J}{2}\left(\frac{S_{2}}{\alpha^{2}}-\frac{S_{1}}{\alpha}\right), G_{2}=\frac{1}{2 \pi} J\left(S_{0}-\frac{S_{2}}{\alpha^{2}}\right), G_{3}=\frac{1}{2 \pi} \frac{J}{2}\left(\frac{S_{2}}{\alpha^{2}}+\frac{S_{1}}{\alpha}\right) \\
H_{1}=H_{2}=H_{3}=0
\end{gathered}
$$

For the discretized form of Eq. 6, the derivatives with respect to $x_{k}(k=1,2)$ of the terms of the G-type and $\mathrm{H}$-type matrices are required and are as follows:

$$
\begin{gathered}
\int_{\star} \frac{\partial w\left(\mathbf{x}_{i}, \mathbf{x}\right)}{\partial x_{k}} N_{j}(\mathbf{x}) \mathrm{d} \star=\left[\frac{\partial G_{1}}{\partial x_{k}}, \frac{\partial G_{2}}{\partial x_{k}}, \frac{\partial G_{3}}{\partial x_{k}}\right]^{T} \\
\int_{\star} \frac{\partial}{\partial x_{k}}\left(\frac{\partial w\left(\mathbf{x}_{i}, \mathbf{x}\right)}{\partial \mathbf{n}}\right) N_{j}(\mathbf{x}) \mathrm{d} \star=\left[\frac{\partial H_{1}}{\partial x_{k}}, \frac{\partial H_{1}}{\partial x_{k}}, \frac{\partial H_{1}}{\partial x_{k}}\right]^{T} \quad \star=\{\Gamma, T\}
\end{gathered}
$$

Again, When a collocation node does not pertain to the integration element, the formulations are:

$$
\begin{array}{ccc}
\frac{\partial G_{1}}{\partial x_{k}}=-\frac{J}{4 \pi}\left(\frac{E_{k 2}}{a^{2}}-\frac{E_{k 1}}{a}\right), & \frac{\partial G_{2}}{\partial x_{k}}=-\frac{J}{4 \pi}\left(E_{k 0}-\frac{E_{k 2}}{a^{2}}\right), & \frac{\partial G_{3}}{\partial x_{k}}=-\frac{J}{4 \pi}\left(\frac{E_{k 2}}{a^{2}}+\frac{E_{k 1}}{a}\right) \\
\frac{\partial H_{1}}{\partial x_{k}}=\frac{1}{4 \pi}\left(\frac{I_{k 2}}{a^{2}}-\frac{I_{k 1}}{a}\right), & \frac{\partial H_{2}}{\partial x_{k}}=\frac{1}{4 \pi}\left(I_{k 0}-\frac{I_{k 2}}{a^{2}}\right), & \frac{\partial H_{3}}{\partial x_{k}}=\frac{1}{4 \pi}\left(\frac{I_{k 2}}{a^{2}}+\frac{I_{k 1}}{a}\right)
\end{array}
$$

Finally, When a collocation node is on the integration element, the formulations are:

$$
\begin{aligned}
& \frac{\partial G_{1}}{\partial x_{k}}=\frac{J D_{k}}{4 \pi \alpha}\left(\frac{T_{2}}{\alpha^{2}}-\frac{T_{1}}{\alpha}\right), \quad \frac{\partial G_{2}}{\partial x_{k}}=\frac{J D_{k}}{2 \pi \alpha}\left(T_{0}-\frac{T_{2}}{\alpha^{2}}\right), \quad \frac{\partial G_{3}}{\partial x_{k}}=\frac{J D_{k}}{4 \pi \alpha}\left(\frac{T_{2}}{\alpha^{2}}+\frac{T_{1}}{\alpha}\right) \\
& \frac{\partial H_{1}}{\partial x_{k}}=\frac{\partial H_{2}}{\partial x_{k}}=\frac{\partial H_{3}}{\partial x_{k}}=0
\end{aligned}
$$

All the formulations above are implemented and tested in the computer code PyDFN3D.

\section{3 | PARALLEL DOMAIN DECOMPOSITION ALGORITHM (DDM)}

In what follows a simple network of intersecting fractures (see Figure 4 is considered. For large DFN problems, in the context of BEM, the direct coupling, i.e. the assembly of a large system of equations, including the compatibility conditions at the interfaces among adjacent domains, may be too computationally demanding. In previous works, parallel DDMs were developed aimed at iteratively solving a series of small dense linear subsystems, rather than a coupled large unsymmetrical sparse linear system 9.1011 .46$. . In DDM, conditions at interfaces are updated at each iteration, until obtaining a solution that fits the specified compatibility condition at each interface [46].

Given $N_{F}$ fractures and $N_{T}$ traces in a DFN, and being $T_{k}$ the set of all traces on fracture $\Omega_{k}, k=1, \ldots, N_{F}$, and $\mathcal{T}$ the set of all the traces in the network, each trace $t \in \mathcal{T}$ is shared by fractures $\Omega_{I}$ and $\Omega_{J}, I \neq J \in\left[1, \ldots, N_{F}\right]$, then a map $\tau$ between each trace and the corresponding pair of fracture indexes: $\tau(t)=\{I, J\}$, being $t=\bar{\Omega}_{I} \cap \bar{\Omega}_{J}$. Compatibility conditions at the traces impose the continuity of pressure and the balance of normal fluxes, which can be 


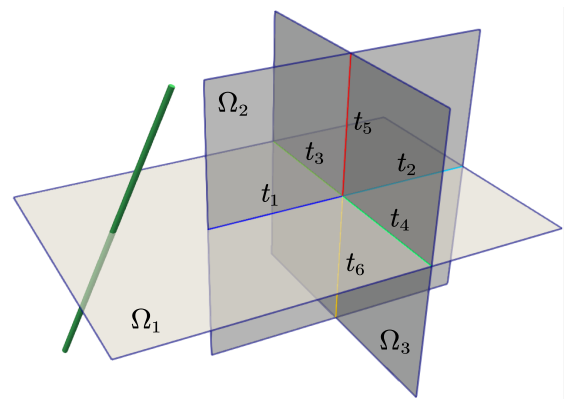

F IG URE 4 Simple DFN with 3 fracture planes, $\Omega_{1}, \Omega_{2}, \Omega_{3}$, and six intersection traces, $t_{1}, t_{2}, t_{3}, t_{4}, t_{5}, t_{6}$

expressed as follows:

$$
\begin{gathered}
\mathbf{q}_{I, t}+\mathbf{q}_{J, t}=0 \\
\mathbf{p}_{I, t}=\mathbf{p}_{I, t}
\end{gathered} \text { on } t,\{I, J\}=\tau(t)
$$

where $q_{I, t}, p_{I, t}$ are the restriction of quantities $q_{I}$ and $p_{I}$, respectively, to trace $t$ on fracture $\Omega_{I}$, for $t \in T_{I}$. Then, the parallel domain decomposition algorithm here used for the resolution of large DFN problems is depicted in Table 1 in which the superscript is used to indicate iteration counter.

TABLE 1 Parallel domain decomposition algorithm

1. set $k=0$ and initial guess $\mathbf{p}_{I, t}^{0}$ on each fracture $\Omega_{I}, I=1, \ldots, N_{F}$ at each intersection trace, $t \in T_{I}$

2. compute $\mathbf{q}_{I}^{0}$ by solving Eq. 10 at each fracture domain $\Omega_{I}, I=1, \ldots, N_{F}$

while $\varepsilon=\sum_{I=1}^{N_{F}} \sum_{t \in T_{I}}\left\|\mathbf{p}_{I, t}^{k+1}-\mathbf{p}_{I, t}^{k}\right\| /\left\|\mathbf{p}_{I, t}^{k}\right\|<$ TOL do

3. update $\mathbf{p}_{I, t}^{k+1}=\mathbf{p}_{I, t}^{k}-\beta^{k}\left(\mathbf{q}_{I, t}^{k}+\mathbf{q}_{J, t}^{k}\right)$, at each intersection trace $t \in \mathcal{T},\{I, J\}=\tau(t)$

4. compute $\mathbf{q}_{I, t}^{k+1}$ by solving Eq. 10 at each sub-domain $\Omega_{I}, I=1, \ldots, N_{F}, t \in T_{I}$

5. $\mathrm{k}=\mathrm{k}+1$

end while

It is to remark that, according to the solution strategy, the compatibility conditions of Eq. 19]are satisfied up to a given tolerance TOL, this quantity being directly associated with the mass flux balance over all intersections. The optimal relaxation parameter $\beta^{k}$ is defined as follows[46]:

$$
\beta^{k}=\frac{\sum_{t \in \mathcal{T}} \epsilon_{p, I, t}^{k} \cdot\left(\epsilon_{q, I, t}^{k}+\epsilon_{q, J, t}^{k}\right)}{\sum_{t \in \mathcal{T}}\left\|\epsilon_{q, I, t}^{k}+\epsilon_{q, J, t}^{k}\right\|^{2}}, \quad\{I, J\}=\tau(t)
$$

with error terms between two iterations $\epsilon_{p, I, t}^{k}=\mathbf{p}_{I, t}^{k+1}-\mathbf{p}_{I, t}^{k}$, and $\epsilon_{q, I, t}^{k}=\mathbf{q}_{I, T_{K}}^{k+1}-\mathbf{q}_{I, t}^{k}$. Clearly, lower values of TOL yeld better flux balance at the traces, at the cost of a larger number of iterations and computational time. A TOL value of $1 \times 10^{-6}-1 \times 10^{-9}$ is used for the following computations. 


\section{4 | NUMERICAL EXAMPLES}

In this section, three numerical examples are presented: the first example is used to verify the proposed technique through a comparison with the exact solution of the steady-state fluid flow in a unit square fracture without internal sources; the other two examples are introduced to demonstrate the robustness and reliability of the technique when dealing with more complex DFN systems.

\section{1 | Unit square problem}

Consider the fluid flow in a unit square $\Omega=\{(x, y) \in(0,1) \times(0,1)\}$ with $\mu=1, k=1$ and $b_{f}=1$. The Darcy problem is considered in $\Omega$ with boundary conditions as in Fig. 5 . The exact solution is known (shown in Fig. 5):

$$
p(x, y)=15 \cos (4 \pi x) \frac{\sinh (4 \pi y)}{\pi \cosh (4 \pi)}
$$

The relative errors of the proposed methods are analyzed by computing the norm errors $e_{p}$ and $e_{\mathbf{u}}$ for $N$ sampling points over the computation domain as below:

$$
e_{p}=\sqrt{\frac{\sum_{l=1}^{N}\left(p_{l, \text { exact }}-p_{l, h}\right)^{2}}{N}}, \quad e_{\mathbf{u}}=\sqrt{\frac{\sum_{l=1}^{N}\left(\mathbf{u}_{l, \text { exact }}-\mathbf{u}_{l, h}\right) \cdot\left(\mathbf{u}_{l, \text { exact }}-\mathbf{u}_{l, h}\right)}{N}}
$$

where $N=1600$ sampling points are uniformly distributed over the domain.
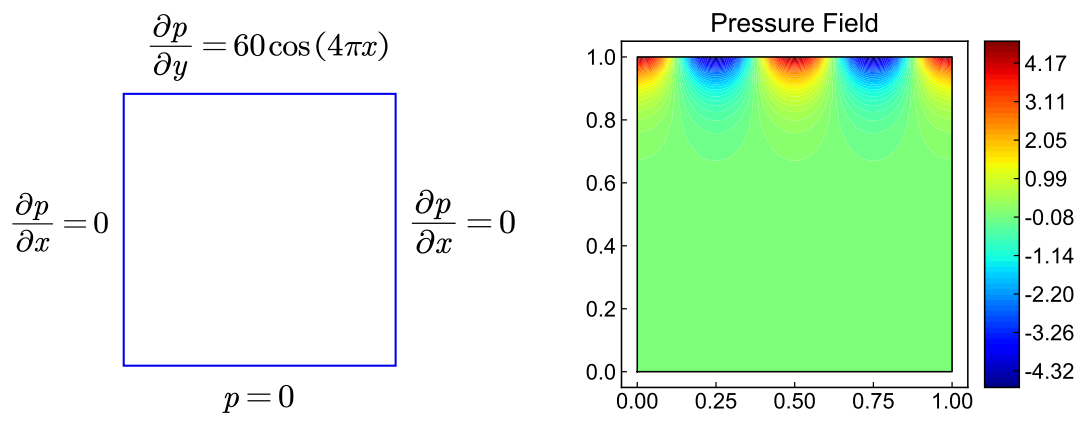

FIGURE 5 Unit square problem: $\Omega$ is a unit square with 3 Neumann boundary conditions and 1 Dirichlet boundary condition

As shown in Fig.6] a reference Finite Element Method (FEM) solution produced by the FEM code COMSOL[21] (using continuous quadratic element) is compared to BEM solutions with constant, discontinuous linear and discontinuous quadratic elements, respectively. The results show that the presented BEM technique can be more accurate than FEM with the same order of basis functions, especially for the velocity values. In this case, the higher-order quadratic BEM produces velocity error of $1.79 \times 10^{-5}$ with the mesh size of 0.16 . While the quadratic FEM produces velocity error of of $1.19 \times 10^{-2}$ with the maximum mesh size of 0.01 . 

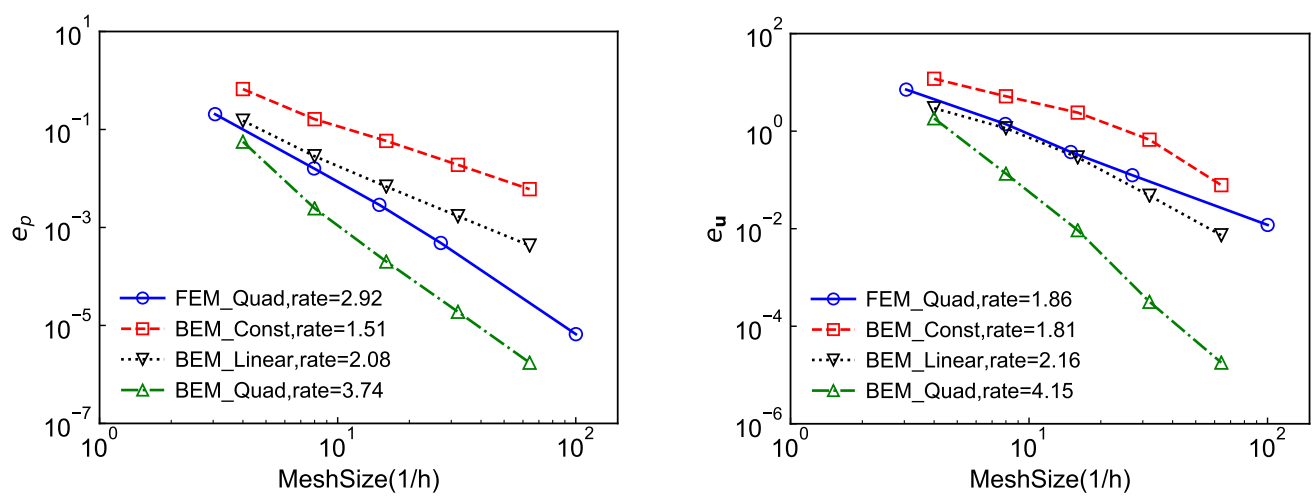

FIGURE 6 Comparison of convergence rate between the BEM solver (PyDFN3D) and the FEM solver (COMSOL) for pressure $p$ and velocity $\mathbf{u}$.

\section{2 | Single fracture domain problem}

In order to further evaluate the robustness and reliability of the proposed technique, a comparison is performed against a FEM solution with the 2nd order accuracy.[26].

The solution for a single fracture domain with one fracture intersection and one well is tested. A uniform pressure of $-5 \mathrm{~Pa}$ is prescribed to the trace; the pressure at the wellbore is fixed at $3 \mathrm{~Pa}$. Fluid viscosity is $2 \mathrm{~Pa} \cdot \mathrm{s}$ and fracture permeability is $3 \mathrm{~m}^{2}$. All other parameters are adopted from the example 1.
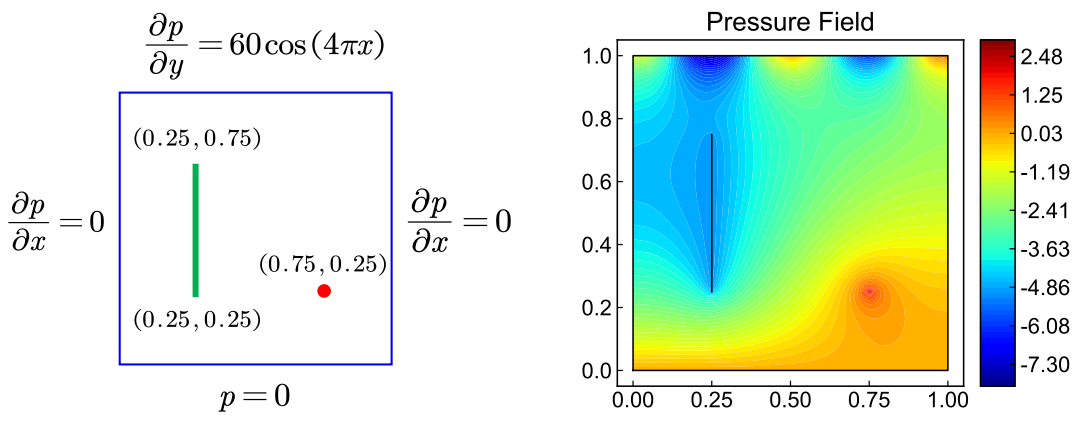

F IGURE 7 Single fracture domain problem: $\Omega$ is a unit square with three edges subjected to a Neumann boundary condition and one edge subjected to a Dirichlet boundary condition; pressures of $-5 \mathrm{~Pa}$ and $3 \mathrm{~Pa}$ are prescribed to the trace and to the wellbore, respectively.

As shown in Figs. 810 the BEM solution using 97 elements is characterized by the same level of accuracy of the FEM solution with 9432 linear elements. Thanks to the exact point source fundamental function adopted, the BEM successfully captures the sharp pressure gradient in the vicinity of wellbore using only one node. 

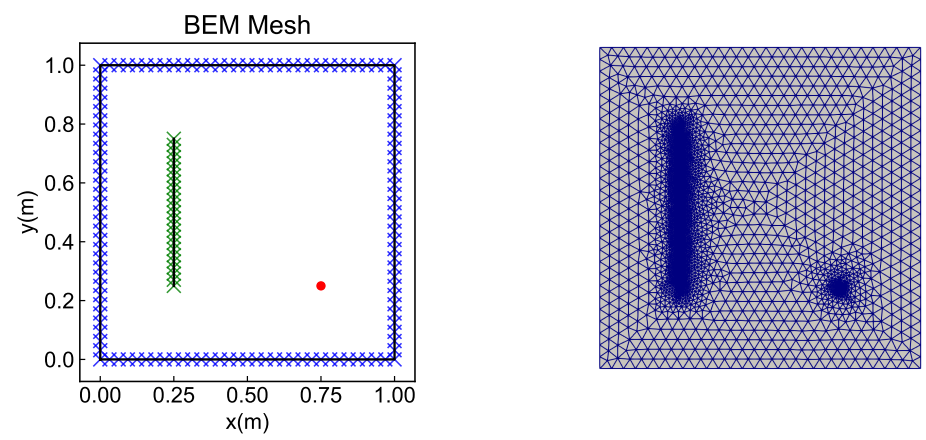

FIGURE 8 Comparison of typical meshes for fracture modeling: BEM refined mesh has 97 elements where FEM LGR mesh has 9432 elements

FIGURE 9 Small scale DFNs domain of Case 3, injector $S_{I n j}$ with pressure constrains of 2 MPa and producer $S_{P r o}$ with pressure constrains of $1 \mathrm{MPa}$
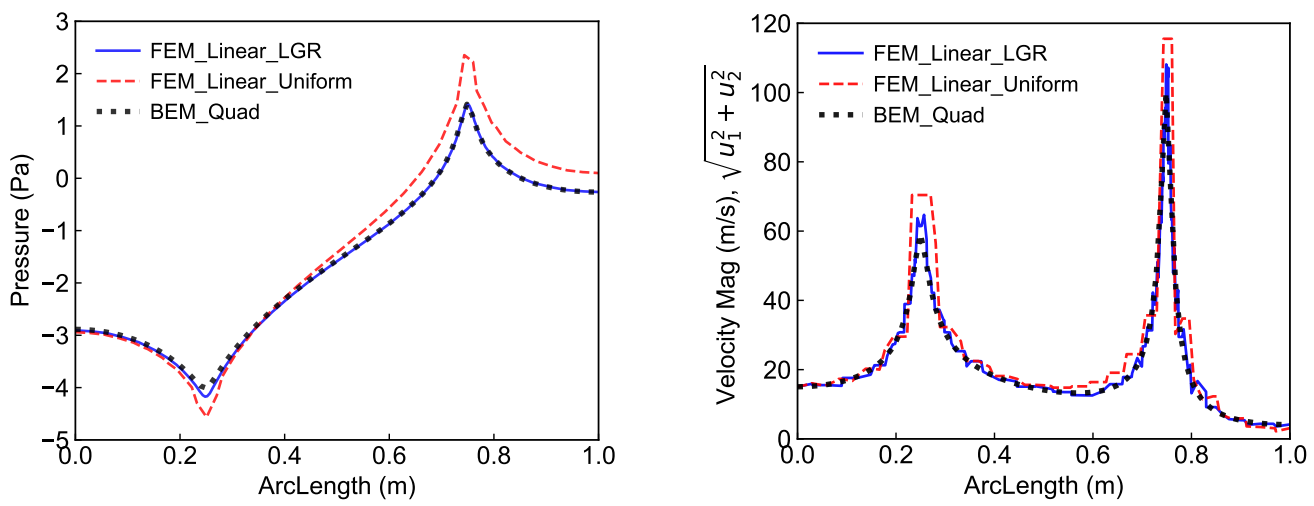

FIGURE 10 Comparison of BEM pressure and discharge solution along $y=0.24$ with the corresponding FEM solution

\subsection{Four-fractures DFN}

With reference to Fig. 11, the steady-state fluid flow in a small DFN consisting of four rectangular fractures is solved. The result of the proposed technique is compared against the result of a FEM model with quadratic elements and a refined mesh. Three horizontal fractures $\Omega_{2-4}$ intersect a vertical fracture $\Omega_{1}$. The injection well $s_{I n j}$ crosses $\Omega_{3}$ and the production well $s_{\text {Pro }}$ crosses two fractures, $\Omega_{2,4}$. Fixed pressure values for injection well and production well are 2 $\mathrm{MPa}$ and $1 \mathrm{MPa}$, respectively. The apertures of the four fractures are set as $0.01 \mathrm{~m}$. Wellbore radius is $0.001 \mathrm{~m}$. The fluid viscosity and fracture permeability are $0.001 \mathrm{~Pa} \cdot \mathrm{s}$ and $3 \times 10^{-10} \mathrm{~m}^{2}$, respectively.

In Fig. 12 the pressure and velocity solutions of the presented BEM technique are compared to the reference FEM solutions. For the BEM solution. only 87 boundary elements resulted sufficient to reach a perfect match with the FEM solution. Owing to DDM, the coupled large unsymmetrical sparse linear system is decomposed into 4 dense linear subsystems. In each iteration, these small subsystems are solved independently. In this example, the proposed 


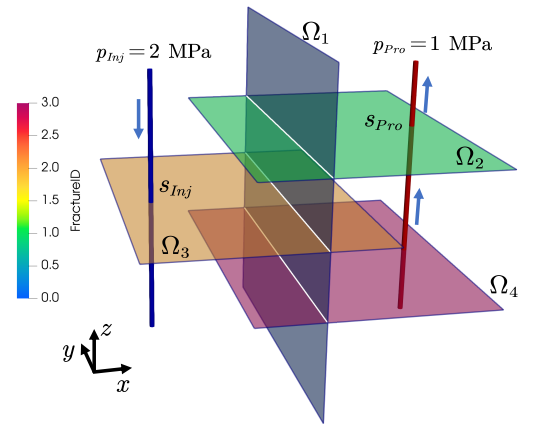

(a) 2D BEM planar model

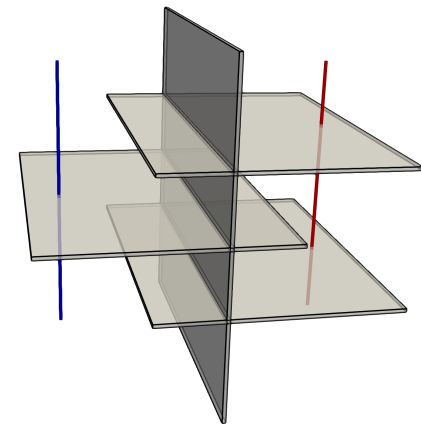

(b) Reference 3D FEM solid model

FIGURE 11 DFNs of four fractures: domain size is $1 \mathrm{~m} \times 1 \mathrm{~m} \times 1 \mathrm{~m}$, wellbore radius is $0.001 \mathrm{~m}$, fracture apertures are constant and equal to $0.01 \mathrm{~m}$; further geometrical details are Appendix $C$

technique furnishes a maximum flux balance error over all traces and wells of $4.2 \times 10^{-9} \mathrm{~m}^{3} / \mathrm{s}$ in 112 iterations. This error can be further reduced by more iterations, however the global flux balance would not change too much. Thus, TOL is set as $1 \times 10^{-9} \mathrm{~m}^{3} / \mathrm{s}$. The calculated flux at the injection well is $13.03 \mathrm{~m}^{3} /$ day, slightly different $(0.2 \%)$ from the one provided by the FEM solution $\left(13.05 \mathrm{~m}^{3} /\right.$ day)

\section{4 | 93-fractures DFN}

The last example consider a 97 -fractures DFN (4 faults and 93 natural fractures) where there are 2 vertical wells, drilled through four faults (Fig. 13a. The 93 natural fractures are randomly generated by the open-source DFN modeling tool ADFNE [1]. Constant pressure values of $15 \mathrm{MPa}$ and $10 \mathrm{MPa}$ are fixed at the injection well and production well, respectively. No flow boundary conditions are prescribed on all fractures. Uniform aperture and permeability are assigned to all the fractures. Based on lab experiments and field observation, fracture apertures and permeability distributions follow specific Probability Density Functions (PDFs), such as power-law and log-normal distribution[14]. As shown in Fig. 13b, the log-normal PDF is adopted to generate the apertures (mean value of -2.1 and standard deviation value of 0.27 are adopted [42]) and the cubic-law $k_{f}=b_{f}{ }^{2} / 12$ is applied to calculate the corresponding permeability [51]. The faults have the same aperture of $3 \times 10^{-4} \mathrm{~m}$ and the same permeability of $7.5 \times 10^{-9} \mathrm{~m}^{2}$.

Fig. 14 a shows the BEM mesh for the fault 0 . Totally 2146 discontinuous quadratic elements ( 23 element per fracture plane) were used in this problem. Fig 14b shows the steady-state pressure solution. 2132 iterations were required to reach a maximum flux balance error over all intersections and wells of $2.6 \times 10^{-6} \mathrm{~m}^{3} / \mathrm{s}$.

It is worthwhile to note that the performance of the parallel DDM algorithm in PyDFN3D could be easily accelerated through a parallel implementation in High Performance Computing (HPC) systems.

\section{5 | CONCLUSIONS}

A novel numerical technique based on the Boundary Element Method (BEM) and with discontinuous quadratic elements and a parallel domain decomposition is presented for solving steady-state fluid flow problems in 3D Discrete Fracture Networks (DFNs). Fast analytical integration formulations were derived to account for singular, nearly-singular and non-singular BEM integrals. Several examples were investigated to verify the accuracy, robustness and applicability of 

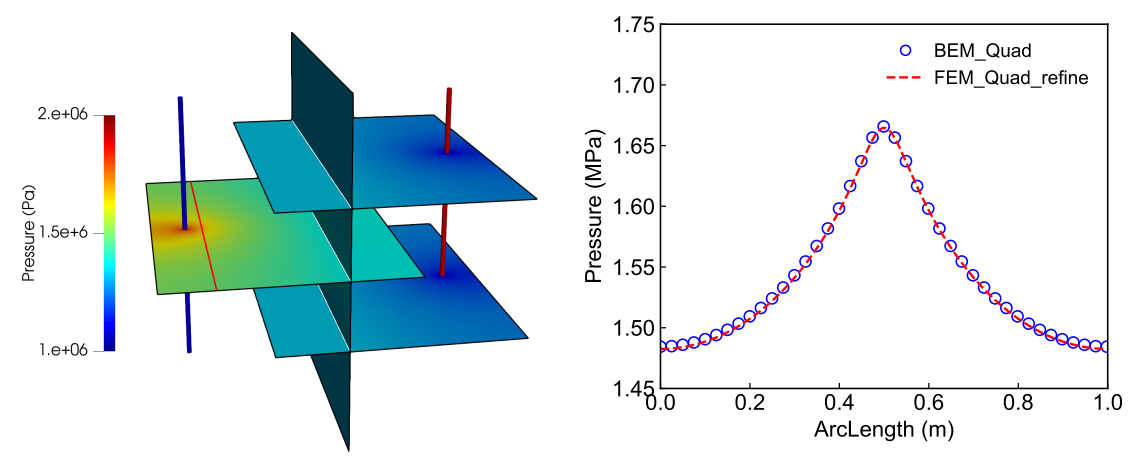

(a) Pressure solution

(b) Pressure $p$ along the red line

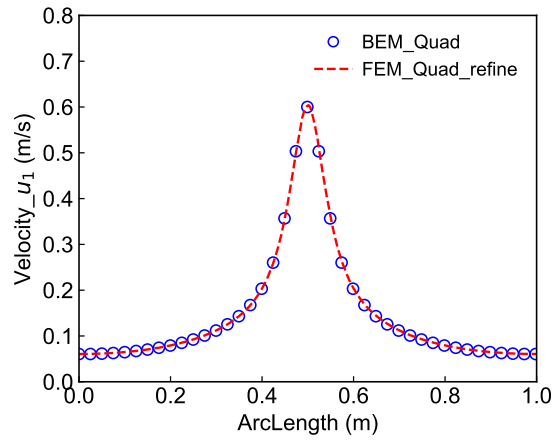

(c) Velocity component $u_{1}$ along the red line

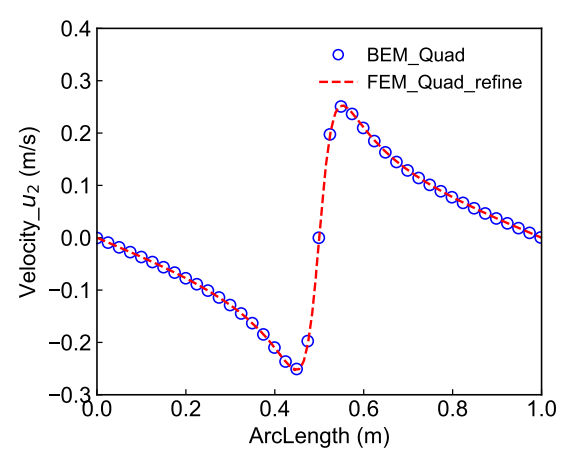

(d) Velocity component $u_{2}$ over the red line

FIGURE 12 Pressure and velocity solution of Case 3. Red line in (a) is the line opf the plots (b)-(d)

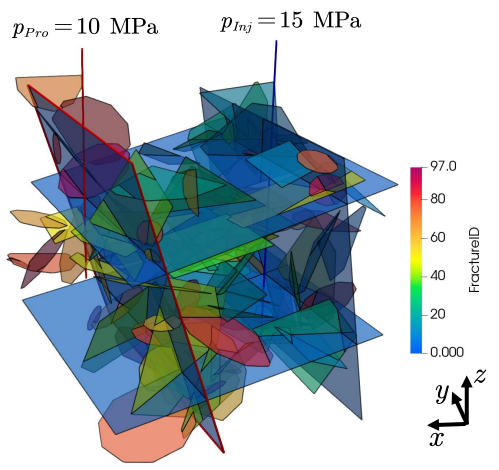

(a) large scale DFNs model where fracture 0 marked in red

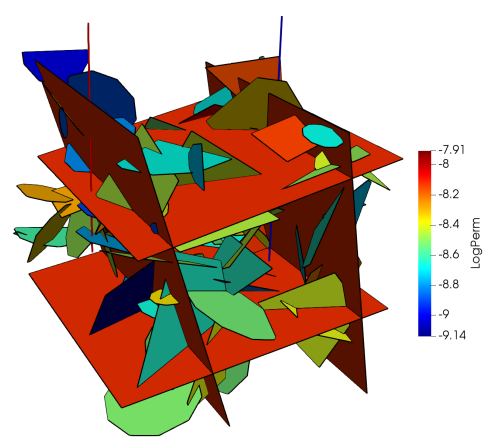

(b) Permeability distribution

FIGURE 13 A DFN with 4 conductive faults and 93 natural fractures, one injection well $S_{I n j}$ with pressure constraint of $15 \mathrm{MPa}$ and one production well $S_{\text {Pro }}$ with pressure constraint of $10 \mathrm{MPa}$ 


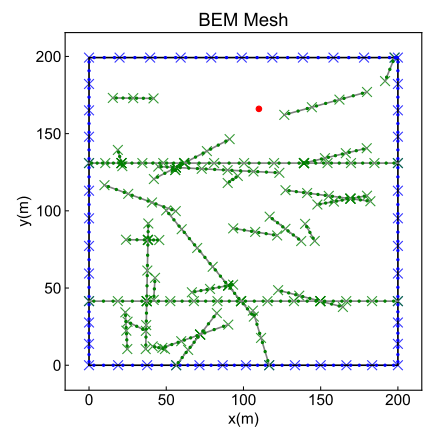

(a) BEM mesh of fault fracture 0

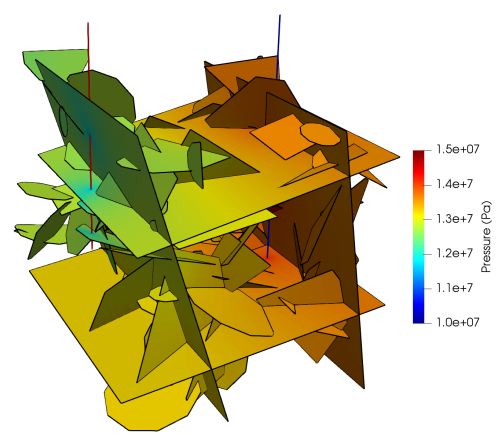

(b) Pressure solution

FIGURE 14 Permeability distribution and pressure solution for the 97-fractures DFN

the proposed method. The following conclusion can be drawn:

- the limitations of a previously proposed BEM technique for 3D DFNs are resolved, including higher computational error due to lower-order piece-wise constant basis function, flux discontinuity at the trace-trace, trace-boundary intersections and fracture-wise homogeneous aperture and permeability;

- the second-order discontinuous quadratic basis functions are employed for the approximation of both pressure and velocity on fracture boundaries and traces; the proposed technique shows fast convergence of pressure and velocity with rates of 3.74 and 4.15 , respectively, much higher than the convergence rates of reference mesh-based method with linear/quadratic element;

- the numerical examples described herein indicate an excellent agreement between the proposed method and a highly-refined reference FEM solution; with the proposed technique much less nodes and elements can be used to achieve similar results by FEM solution;

- the use of the point source function results highly effective for the simulation of the sharp pressure gradients in the near-field of a wellbore;

- the companion parallel DDM algorithm shows good convergence properties, although the number of iterations could be reduced.

It is intention of the authors to implement in the future the proposed method in a parallel code, also resorting to other DDM algorihtms.

\section{ACKNOWLEDGMENTS}

This research was supported by Board of Regents of the State of Louisiana (LEQSF(2017-20)-RD-A-20). The authors express their appreciation to Dr. Boyun Guo (University of Louisiana at Lafayette) and Tianqi Ma (Fudan University) for their support. S.B., S.P. and S.S. acknowledge the support of INdAM-GNCS and acknowledge that the present research has been partially supported by MIUR grant Dipartimenti di Eccellenza 2018-2022 (E11G18000350001) and MIUR project PRIN 201744KLJL_004. 


\section{References}

[1] Y.F. Alghalandis, Adfne: Open source software for discrete fracture network engineering, two and three dimensional applications, Comput. Geosciences 102 (2017), 1-11.

[2] J. Andersson and B. Dverstorp, Conditional simulations of fluid flow in three-dimensional networks of discrete fractures, Water Resources Res. 23 (1987), 1876-1886.

[3] C.A. Andresen, A. Hansen, R. Le Goc, P. Davy, and S.M. Hope, Topology of fracture networks, Frontiers Phys. 1 (2013), 7.

[4] P.F. Antonietti, L. Formaggia, A. Scotti, M. Verani, and N. Verzott, Mimetic finite difference approximation of flows in fractured porous media, ESAIM: Math. Modelling Numer. Anal. 50 (2016), 809-832.

[5] M.F. Benedetto, S. Berrone, and S. Scialò, A globally conforming method for solving flow in discrete fracture networks using the virtual element method, Finite Elements Anal. Design 109 (2016), 23-36.

[6] M.F. Benedetto, A. Borio, and S. Scialo, Mixed virtual elements for discrete fracture network simulations, Finite Elements Anal. Design 134 (2017), 55-67.

[7] S. Berrone, A. Borio, and F. Vicini, Reliable a posteriori mesh adaptivity in discrete fracture network flow simulations, Comput. Methods Appl. Mechanics Eng. 354 (2019), 904-931.

[8] S. Berrone, C. Fidelibus, S. Pieraccini, S. Scialó, and F. Vicini, Unsteady advection-diffusion simulations in complex discrete fracture networks with an optimization approach, J. Hydrology 566 (2018), 332-345.

[9] S. Berrone, S. Pieraccini, and S. Scialo, A pde-constrained optimization formulation for discrete fracture network flows, SIAM J. Sci. Comput. 35 (2013), B487-B510.

[10] S. Berrone, S. Pieraccini, and S. Scialò, An optimization approach for large scale simulations of discrete fracture network flows, J. Comput. Phys. 256 (2014), 838-853.

[11] S. Berrone, S. Pieraccini, S. Scialo, and F. Vicini, A parallel solver for large scale dfn flow simulations, SIAM J. Sci. Comput. 37 (2015), C285-C306.

[12] S. Berrone, S. Scialo, and F. Vicini, Parallel meshing, discretization, and computation of flow in massive discrete fracture networks, SIAM J. Sci. Comput. 41 (2019), C317-C338.

[13] C.A. Brebbia and J. Dominguez, Boundary elements: an introductory course, WIT press, 1994.

[14] M.C. Cacas, E. Ledoux, G. de Marsily, B. Tillie, A. Barbreau, E. Durand, B. Feuga, and P. Peaudecerf, Modeling fracture flow with a stochastic discrete fracture network: calibration and validation: 1. the flow model, Water Resources Res. 26 (1990), 479-489.

[15] F. Chave, D.A. Di Pietro, and L. Formaggia, A hybrid high-order method for darcy flows in fractured porous media, SIAM J. Sci. Comput. 40 (2018), A1063-A1094.

[16] Z. Chen, Reservoir simulation: mathematical techniques in oil recovery Vol. 77, Siam, 2007.

[17] Z. Chen, X. Liao, K. Sepehrnoori, W. Yu, et al., A semianalytical model for pressure-transient analysis of fractured wells in unconventional plays with arbitrarily distributed discrete fractures, SPE J. (2018).

[18] Z. Chen, X. Liao, X. Zhao, X. Dou, and L. Zhu, Performance of horizontal wells with fracture networks in shale gas formation, J. Petroleum Sci. Eng. 133 (2015), 646-664.

[19] Z. Chen, X. Liao, X. Zhao, X. Dou, L. Zhu, L. Sanbo, et al., A finite-conductivity horizontal-well model for pressure-transient analysis in multiple-fractured horizontal wells, SPE J. 22 (2017), 1-112. 
[20] Z. Chen, X. Liao, X. Zhao, and L. Zhu, Influence of magnitude and permeability of fracture networks on behaviors of vertical shale gas wells by a free-simulator approach, J. Petroleum Sci. Eng. 147 (2016), 261-272.

[21] COMSOL Inc., Multiphysics reference guide for comsol 5.2.

[22] W. Dershowitz and C. Fidelibus, Derivation of equivalent pipe network analogues for three-dimensional discrete fracture networks by the boundary element method, Water Resources Res. 35 (1999), 2685-2691.

[23] Y. Dong, Y. Fu, T.C.J. Yeh, Y.L. Wang, Y. Zha, L. Wang, and Y. Hao, Equivalence of discrete fracture network and porous media models by hydraulic tomography, Water Resources Res. ().

[24] M. Fratantonio and J. Rencis, Exact boundary element integrations for two-dimensional laplace equation, Eng. Anal. with Boundary Elements 24 (2000), 325-342.

[25] A. Fumagalli and E. Keilegavlen, Dual virtual element method for discrete fractures networks, SIAM J. Sci. Comput. 40 (2018), B228-B258.

[26] A. Fumagalli, E. Keilegavlen, and S. Scialò, Conforming, non-conforming and non-matching discretization couplings in discrete fracture network simulations, J. Comput. Phys. 376 (2019), 694-712.

[27] Y. Gu, Q. Hua, W. Chen, and C. Zhang, Numerical evaluation of nearly hyper-singular integrals in the boundary element analysis, Comput. Structures 167 (2016), 15-23.

[28] J.D. Hyman, A. Hagberg, G. Srinivasan, J. Mohd-Yusof, and H. Viswanathan, Predictions of first passage times in sparse discrete fracture networks using graph-based reductions, Physical Review E 96 (2017), 013304.

[29] J.D. Hyman, A. Hagberg, G. Srinivasan, J. Mohd-Yusof, and H. Viswanathan, Predictions of first passage times in sparse discrete fracture networks using graph-based reductions, Physical Review E 96 (2017), 013304.

[30] J.D. Hyman, S. Karra, N. Makedonska, C.W. Gable, S.L. Painter, and H.S. Viswanathan, dfnworks: A discrete fracture network framework for modeling subsurface flow and transport, Comput. Geosciences 84 (2015), 10-19.

[31] M. Iding and P. Ringrose, Evaluating the impact of fractures on the performance of the in salah co2 storage site, Int. J. Greenhouse Gas Control 4 (2010), 242-248.

[32] S. Karra, N. Makedonska, H.S. Viswanathan, S.L. Painter, and J.D. Hyman, Effect of advective flow in fractures and matrix diffusion on natural gas production, Water Resources Res. 51 (2015), 8646-8657.

[33] J.T. Katsikadelis, The boundary element method for engineers and scientists: theory and applications, Academic Press, 2016.

[34] S.H. Lee, M. Lough, and C. Jensen, Hierarchical modeling of flow in naturally fractured formations with multiple length scales, Water resources research 37 (2001), 443-455.

[35] V. Lenti and C. Fidelibus, A bem solution of steady-state flow problems in discrete fracture networks with minimization of core storage, Comput. geosciences 29 (2003), 1183-1190.

[36] Y. Liu and N. Nishimura, The fast multipole boundary element method for potential problems: a tutorial, Eng. Anal. with Boundary Elements 30 (2006), 371-381.

[37] M. McClure, M. Babazadeh, S. Shiozawa, J. Huang, et al., Fully coupled hydromechanical simulation of hydraulic fracturing in three-dimensional discrete fracture networks, SPE Hydraulic Fracturing Technology Conference, 2015.

[38] A. Meurer, C.P. Smith, M. Paprocki, O. Čertík, S.B. Kirpichev, M. Rocklin, A. Kumar, S. Ivanov, J.K. Moore, S. Singh, T. Rathnayake, S. Vig, B.E. Granger, R.P. Muller, F. Bonazzi, H. Gupta, S. Vats, F. Johansson, F. Pedregosa, M.J. Curry, A.R. Terrel, v. Roučka, A. Saboo, I. Fernando, S. Kulal, R. Cimrman, and A. Scopatz, Sympy: symbolic computing in python, PeerJ Comput. Sci. 3 (Jan. 2017), e103. 
[39] M. Sahimi, Flow and transport in porous media and fractured rock: from classical methods to modern approaches, John Wiley \& Sons, 2011.

[40] A.M. Shapiro and J. Andersson, Steady state fluid response in fractured rock: a boundary element solution for a coupled, discrete fracture continuum model, Water Resources Res. 19 (1983), 959-969.

[41] A.M. Shapiro and J. Andersson, Simulation of steady-state flow in three-dimensional fracture networks using the boundaryelement method, Advances water resources 8 (1985), 106-110.

[42] D.T. Snow, The frequency and apertures of fractures in rock, International journal of Rock mechanics and Mining sciences \& Geomechanics Abstracts, Vol. 7, 1970, pp. 23-40.

[43] Z.x. Sun, X. Zhang, Y. Xu, J. Yao, H.x. Wang, S. Lv, Z.I. Sun, Y. Huang, M.y. Cai, and X. Huang, Numerical simulation of the heat extraction in egs with thermal-hydraulic-mechanical coupling method based on discrete fractures model, Energy 120 (2017), 20-33.

[44] M. Tanaka, V. Sladek, and J. Sladek, Regularization techniques applied to boundary element methods, Appl. Mechanics Reviews 47 (1994), 457-499.

[45] B. Wang, Y. Feng, J. Du, Y. Wang, S. Wang, R. Yang, et al., An embedded grid-free approach for near-wellbore streamline simulation, SPE J. 23 (2018), 567-588.

[46] B. Wang, Y. Feng, S. Pieraccini, S. Scialò, and C. Fidelibus, Iterative coupling algorithms for large multidomain problems with the boundary element method, Int. J. Numer. Methods Eng. 117 (2019), 1-14.

[47] C. Xu, C. Fidelibus, P. Dowd, Z. Wang, and Z. Tian, An iterative procedure for the simulation of the steady-state fluid flow in rock fracture networks, Eng. geology 242 (2018), 160-168.

[48] R. Yang, Z. Huang, W. Yu, G. Li, W. Ren, L. Zuo, X. Tan, K. Sepehrnoori, S. Tian, and M. Sheng, A comprehensive model for real gas transport in shale formations with complex non-planar fracture networks, Sci. reports 6 (2016), 36673.

[49] X. Zhang and X. An, Exact integration and its application in adaptive boundary element analysis of two-dimensional potential problems, Commun. Numer. Methods Eng. 24 (2008), 1239-1250.

[50] X. Zhang and X. Zhang, Exact integration in the boundary element method for two-dimensional elastostatic problems, Eng. analysis with boundary elements 27 (2003), 987-997.

[51] R.W. Zimmerman and G.S. Bodvarsson, Hydraulic conductivity of rock fractures, Transport porous media 23 (1996), 1-30.

\section{A | DERIVATION OF THE BIE}

The governing equation for the fluid flow in a fracture is obtained in Eq.4:

$$
-\frac{k b_{f}}{\mu} \nabla^{2} p=\sum_{t \in T} \delta_{t, q_{t}}+\sum_{s \in S} q_{s} \delta_{s}
$$

A weighted residual statement for Eq.4 is as follows:

$$
-\frac{k b_{f}}{\mu}\left(\int_{\Omega} \nabla \cdot(\nabla p w) \mathrm{d} \Omega-\int_{\Omega} \nabla p \cdot \nabla w \mathrm{~d} \Omega\right)=\sum_{t \in T} \int_{t} q_{t}(\gamma) w(\gamma) d \gamma+\sum_{s \in S} q_{s} w\left(\mathbf{x}_{s}\right)
$$


where $w$ is a weighting function. It can be re-written as follows:

$$
-\frac{k b_{f}}{\mu}\left(\int_{\Omega} \nabla \cdot(w \nabla p) \mathrm{d} \Omega-\int_{\Omega} \nabla \cdot(p \nabla w) \mathrm{d} \Omega+\int_{\Omega} \nabla \cdot(\nabla w) p \mathrm{~d} \Omega\right)=\sum_{t \in T} \int_{t} q_{t}(\mathbf{x}) w \mathrm{~d} t+\sum_{s \in S} q_{s} w\left(\mathbf{x}_{s}\right) .
$$

By formally using twice the divergence theorem, once for $\int_{\Omega} \nabla \cdot(\nabla p w) d \Omega=\int_{\Gamma} w \nabla p \cdot \mathbf{n} d \Gamma$, and then $\int_{\Omega} \nabla \cdot(p \nabla w) d \Omega=$ $\int_{\Gamma} p \nabla w \cdot \mathbf{n} d \Gamma$ one obtains:

$$
-\frac{k b_{f}}{\mu}\left(\int_{\Gamma} w \nabla p \cdot \mathrm{nd} \Gamma-\int_{\Gamma} p \nabla w \cdot \mathrm{nd} \Gamma+\int_{\Omega} \nabla \cdot(\nabla w) p \mathrm{~d} \Omega\right)=\sum_{t \in T} \int_{t} q_{t}(\mathbf{x}) w \mathrm{~d} t+\sum_{s \in S} q_{s} w\left(\mathbf{x}_{s}\right)
$$

which can be compactly rewritten as:

$$
-\frac{k b_{f}}{\mu}\left(\int_{\Gamma} w \frac{\partial p}{\partial \mathbf{n}} \mathrm{d} \Gamma-\int_{\Gamma} p \frac{\partial w}{\partial \mathbf{n}} \mathrm{d} \Gamma+\int_{\Omega} \nabla \cdot(\nabla w) p \mathrm{~d} \Omega\right)=\sum_{t \in T} \int_{t} q_{t}(\mathbf{x}) w \mathrm{~d} t+\sum_{s \in S} q_{s} w\left(\mathbf{x}_{s}\right)
$$

If the weighting function $w$ is assumed equal to the fundamental solution of Laplacian operator in $2 \mathrm{D}, \operatorname{say} w(\mathbf{x}, \mathbf{y})$, such that $\int_{\Omega} \nabla_{\mathbf{x}} \cdot\left(\nabla_{\mathbf{x}} w(\mathbf{x}, \mathbf{y})\right) d \mathbf{x}=\delta_{\mathbf{y}}$, then Eq A.5 becomes:

$$
-\frac{k b_{f}}{\mu}\left(\int_{\Gamma} w \frac{\partial p}{\partial \mathbf{n}} \mathrm{d} \Gamma-\int_{\Gamma} p \frac{\partial w}{\partial \mathbf{n}} \mathrm{d} \Gamma-p\right)=\sum_{t \in T} \int_{t} q_{t}(\mathbf{x}) w d t+\sum_{s \in S} q_{s} w\left(\mathbf{x}_{s}\right)
$$

The BIE is finally derived from Eq A.6] using the concept of half circle or half spherical problem [13] as:

$$
\begin{array}{r}
-\frac{k b_{f}}{\mu}\left(c\left(\mathbf{x}_{i}\right) p\left(\mathbf{x}_{i}\right)+\int_{\Gamma} p(\mathbf{x}) \frac{\partial w\left(\mathbf{x}_{i}, \mathbf{x}\right)}{\partial \mathbf{n}} \mathrm{d} \Gamma\right)=-\frac{k b_{f}}{\mu} \int_{\Gamma} \frac{\partial p}{\partial \mathbf{n}}(\mathbf{x}) w\left(\mathbf{x}_{i}, \mathbf{x}\right) \mathrm{d} \Gamma \\
-\sum_{t \in T} \int_{t} q_{t}(\mathbf{x}) w\left(\mathbf{x}_{i}, \mathbf{x}\right) \mathrm{d} t-\sum_{s \in S} q_{s} w\left(\mathbf{x}_{i}, \mathbf{x}_{s}\right)
\end{array}
$$

where $\mathbf{x}_{i}$ denotes the source point, and $c\left(\mathbf{x}_{i}\right)=1$ if $\mathbf{x}_{i}$ is located inside the domain or $c\left(\mathbf{x}_{i}\right)=\frac{1}{2}$ if $\mathbf{x}_{i}$ is located on the boundary, if the boundary is smooth [13].

\section{B | ANALYTICAL INTEGRATIONS}

By assuming that a quadratic BEM element is a straight line, as shown in Figs. [2-3], the integrals in 13 can be analytically calculated and the results of the integration subsequently reported in 2.3 by using the integral quantities reported in what follows.

The integrals depend on the locations of the two extreme nodes $\left(x_{1}(1), x_{2}(1)\right)$ and $\left(x_{1}(3), x_{2}(3)\right)$ of the integration element and of the collocation node $\left(x_{1 i}, x_{2 i}\right)$. According to the method proposed by [50, 49], the following geometrical 
quantities are defined first:

$$
\begin{array}{cl}
D_{1}=\frac{x_{1}(3)-x_{1}(1)}{2}, & D_{2}=\frac{x_{2}(3)-x_{2}(1)}{2} \\
C_{1}=\frac{x_{1}(3)+x_{1}(1)}{2}-x_{1 i}, & C_{2}=\frac{x_{2}(3)+x_{2}(1)}{2}-x_{2 i} \\
a=D_{1}^{2}+D_{2}^{2}, \quad b=2\left(D_{1} C_{1}+D_{2} C_{2}\right), & c=C_{1}^{2}+C_{2}^{2}, \quad e=C_{1} D_{2}-C_{2} D_{1} \\
J=L / 2 &
\end{array}
$$

where $J$ is the Jacobian of a straight element and $L$ is the length of the element.

Using the constants defined above, the following integrals are solved:

$$
F_{n}=\int_{-1}^{1} \frac{\xi^{n}}{a \xi^{2}+b \xi+c} \mathrm{~d} \xi, \quad A_{n}=\int_{-1}^{1} \xi^{n} \ln \left(a \xi^{2}+b \xi+c\right) \mathrm{d} \xi, \quad S_{n}=\int_{-1}^{1} \xi^{n} \ln \frac{L}{2}\left|\xi-\xi_{i}\right| \mathrm{d} \xi
$$

With the help of the symbolic mathematics tool SymPy [38], the solutions of the above integrals can be found:

- Integrals $F_{n}$ :

$$
F_{0}=\left\{\begin{array}{cc}
\frac{2}{\sqrt{4 a c-b^{2}}}\left(\arctan \frac{2 a+b}{\sqrt{4 a c-b^{2}}}-\arctan \frac{-2 a+b}{\sqrt{4 a c-b^{2}}}\right), & \text { for } \sqrt{4 a c-b^{2}}>0 \\
\frac{2}{b-2 a}-\frac{2}{b+2 a} & \text { for } \sqrt{4 a c-b^{2}}=0 \\
F_{1}=\frac{1}{2 a} \ln \frac{a+b+c}{a-b+c}-\frac{b}{2 a} F_{0}, & \\
F_{2} & =\frac{2}{a}-\frac{c}{a} F_{0}-\frac{b}{a} F_{1} \\
F_{3} & =-\frac{c}{a} F_{1}-\frac{b}{a} F_{2}, \\
F_{4} & =\frac{2}{3 a}-\frac{c}{a} F_{2}-\frac{b}{a} F_{3}, \\
F_{5} & =-\frac{c}{a} F_{3}-\frac{b}{a} F_{4}
\end{array}\right.
$$

- Integrals $A_{n}$ :

$$
\begin{gathered}
A_{0}=\ln \left[(a+c)^{2}-b^{2}\right]-2 a F_{2}-b F_{1} \\
A_{1}=\frac{1}{2} \ln \frac{a+b+c}{a-b+c}-a F_{3}-\frac{1}{2} F_{2} \\
A_{2}=\frac{1}{3} \ln \left[(a+c)^{2}-b^{2}\right]-\frac{2}{3} a F_{4}-\frac{1}{3} b F_{3}
\end{gathered}
$$

- Integrals $S_{n}$ :

$$
\begin{gathered}
S_{0}=\ln \frac{L}{2}\left(1+\xi_{i}\right)+\ln \frac{L}{2}\left(1-\xi_{i}\right)-\xi_{i} \ln \frac{1+\xi_{i}}{1-\xi_{i}}-2 \\
S_{1}=\frac{1}{2}\left(1-\xi_{i}^{2}\right) \ln \frac{1+\xi_{i}}{1-\xi_{i}}-\xi_{i} \\
S_{2}=\left(1+\xi_{i}\right)^{3}\left[\frac{1}{3} \ln \frac{L}{2}\left(1+\xi_{i}\right)-\frac{1}{9}\right]+\left(1-\xi_{i}\right)^{3}\left[\frac{1}{3} \ln \frac{L}{2}\left(1-\xi_{i}\right)-\frac{1}{9}\right]+2 \xi_{i} S_{1}-\xi_{i}^{2} S_{0}
\end{gathered}
$$

where $\xi_{i}$ is the local coordinate of the collocation node when it lies on the integration element. 
The following integrals are also solved:

$$
G_{n}=\int_{-1}^{1} \frac{\xi^{n}}{\left(a \xi^{2}+b \xi+c\right)^{2}} \mathrm{~d} \xi, \quad T_{n}=\int_{-1}^{1} \frac{\xi^{n}}{\left|\xi-\xi_{i}\right|} \mathrm{d} \xi
$$

In formula:

- Integrals $G_{n}$ :

$$
\begin{aligned}
& G_{1}= \begin{cases}\frac{-2 c+b}{\sqrt{4 a c-b^{2}}(a+b+c)}-\frac{2 c-b}{\sqrt{4 a c-b^{2}}(a-b+c)}-\frac{b}{\sqrt{4 a c-b^{2}}} F_{0} & \text { for } \sqrt{4 a c-b^{2}}>0 \\
-\left[\frac{8 a}{3(b-2 a)^{3}}+\frac{8 a}{3(b+2 a)^{3}}\right]-\frac{2}{3}\left[\frac{1}{(2 a+b)^{2}}-\frac{1}{(-2 a+b)^{2}}\right], & \text { for } \sqrt{4 a c-b^{2}}=0\end{cases} \\
& G_{2}=-\frac{1}{a(a-b+c)}-\frac{1}{a(a+b+c)}+\frac{c}{a} G_{0} \\
& G_{3}=\frac{1}{2 a^{2}}\left[\ln \frac{a+b+c}{a-b+c}-3 a b G_{2}-\left(2 a c+b^{2}\right) G_{1}-b c G_{0}\right]
\end{aligned}
$$

where the quantity $G_{0}$ is as follows:

$$
G_{0}= \begin{cases}\frac{2 a+b}{\sqrt{4 a c-b^{2}}(a+b+c)}-\frac{-2 a+b}{\sqrt{4 a c-b^{2}}(a-b+c)}+\frac{2 a}{\sqrt{4 a c-b^{2}}} F_{0}, & \text { for } \sqrt{4 a c-b^{2}}>0 \\ \frac{8 a}{3(b-2 a)^{3}}-\frac{8 a}{3(b+2 a)^{3}} & \text { for } \sqrt{4 a c-b^{2}}=0\end{cases}
$$

- Integrals $T_{n}$ :

$$
T_{0}=\ln \frac{1+\xi_{i}}{1-\xi_{i}}, \quad T_{1}=\xi_{i} \ln \frac{1+\xi_{i}}{1-\xi_{i}}+2, \quad T_{2}=\xi_{i}^{2} \ln \frac{1+\xi_{i}}{1-\xi_{i}}+2 \xi_{i}
$$

Finally, the quantities $E_{1 n}, E_{2 n}$ and $I_{1 n}, I_{2 n}(n=0,1,2)$ are defined as follows:

$$
\begin{gathered}
E_{1 n}=C_{1} F_{n}+D_{1} F_{n+1}, \\
E_{2 n}=C_{2} F_{n}+D_{2} F_{n+1}, \\
I_{1 n}=2 e\left(C_{1} G_{n}+D_{1} G_{i+1}\right)-D_{2} F_{i}, \\
I_{2 n}=2 e\left(C_{2} G_{i}+D_{2} G_{i+1}\right)+D_{1} F_{i}
\end{gathered}
$$




\section{I GEOMETRIC PARAMETERS FOR THE FOUR-FRACTURES DFN}

TAB LE C 1 Geometric parameters table for the four fracture planes, two wells and red sampling line of Case 3

\begin{tabular}{lcccc} 
Object & Coordinates & & Dimension (DX,DY,DZ) & Shape \\
$\Omega_{1}$ & $(0.0,-0.5,-0.5)$ & $-(0.0,0.5,0.5)$ & $(0.0,1.0,1.0)$ & Rectangle \\
$\Omega_{2}$ & $(-0.2,-0.5,0.2)$ & $-(0.5,0.5,0.2)$ & $(0.7,1.0,0.0)$ & Rectangle \\
$\Omega_{3}$ & $(-0.5,-0.5,0.0)$ & $-(0.2,0.5,0.0)$ & $(0.7,1.0,0.0)$ & Rectangle \\
$\Omega_{4}$ & $(-0.2,-0.5,-0.2)$ & $-(0.5,0.5,-0.2)$ & $(0.7,1.0,0.0)$ & Rectangle \\
$S_{I n j}$ & $(-0.4,0.0,-0.4)$ & $-(-0.4,0.0,0.4)$ & $(0.0,0.0,0.8)$ & Line segment \\
$S_{\text {Pro }}$ & $(0.4,0.0,-0.4)$ & $-(0.4,0.0,0.4)$ & $(0.0,0.0,0.8)$ & Line segment \\
Red Line & $(-0.35,-0.5,0.0)$ & $-(-0.35,0.5,0.0)$ & $(0.0,1.0,0.0)$ & Line segment \\
\hline
\end{tabular}

$\mathrm{DX}, \mathrm{DY}, \mathrm{DZ}$, the length of object in $\mathrm{x}, \mathrm{y}, \mathrm{z}$ directions 